\title{
SKEW POLYNOMIAL RINGS SATISFYING $R$-BND PROPERTY
}

\author{
REFAAT M. SALEM
}

\begin{abstract}
In this paper we show that, a prime right Noetherian ring $A$ satisfies $T(A)=n<\infty$ iff $A[x, \sigma]$ satisfies $r$-Bnd $(n+1)$.
\end{abstract}

\section{O. Introduction}

In [6] Robson has found a relation between the Krull dimension (in the sense of Rentschler, [5]) and the upper bound of the number of generators of right ideals in polynomial rings over simple right Noetherian rings. Also Stafford in [8] studied the relation between a ring $A$ and its polynomial ring $A[x]$ if one of them satisfies r-Bnd property. Here we extend Robson's result to the Ore extension over simple right Noetherian rings and study the relation between the two properties $T(A)=n<\infty$ and the $r$ - $\operatorname{Bnd}(n)$ of the skew polynomial ring $A[x, \sigma]$.

\section{Definitions and Basic Concepts}

All rings here are with identity and all modules are unitary. $\mathrm{A}$ ring $A$ is said to be $T(A)=n<\infty$, if every finitely generated torsion right $A$-module can be generated by $n$ elements. The ring $A$ (the module $M_{A}$ ) is said to be $r$-band $(n)$, if every right ideal of $A$ (submodule of $M_{A}$ ) can be generated by $n$ elements. A right $A$-module $M$ is said to be completely faithful, if each nonzero subfactor module is faithful, it is clear that each module over simple rings is completely

Received. June 24, 1991. 
faithful. Consider the Ore extension ring of $A$, that is the ring $\mathbb{R}=A[x, \sigma, \delta]$, where $\sigma$ is an automorphism of $A$ and $\delta$ is a $\sigma$-derivation of $A$, where addition is component wise and multiplication is given as

$$
(a b) x=x \sigma(a b)+\delta(a b) \text { and } \delta(a b)=\sigma(a) \quad \delta(b)+a \delta(b) .
$$

The right ideal $I$ of $A$ is called $\sigma$-ideal if $\sigma(I) \subseteq I$, it is well known that if $A$ is right Noetherian ring, then $\sigma(I)=I$. The ideal $I$ is called $\sigma$-prime ideal of $A$, if whenever $J, K$ are $\sigma$-ideals of $A$ such that $J K \subseteq \mathbb{I}$, then $j \subseteq \mathbb{I}$ or $\mathbb{K} \subseteq \mathbb{I}$. $A$ is called $\sigma$-prime ring if (0) is a $\sigma$-prime ideal of $A$. The ideal $I$ is called $\sigma$-maximal of $A$, if there is no proper $\sigma$-ideal $J$ such that $I \subset J \subset A$.

\section{Preliminary Results and Remarks}

1. Let $R=A[x, \sigma, \delta]$, where $A$ is any ring, $\sigma$ is an automorphism of $A$ and $\delta$ is a $\sigma$-derivation of $A$. Let $S$ be a multiplicative set of regular elements of $A$, such that $\sigma(S) \subseteq S$ and $S$ satisfies the right Ore condition. Let $Q=A S^{-1}$, then

i) $\sigma$ and $\delta$ can be extended in a unique manner to an automorphism $\sigma^{\prime}$ of $Q$ and to a, $\sigma^{\prime}$-derivation $\delta^{\prime}$ of $Q$.

ii) $S$ is the multiplicative set of regular elements of $\mathbb{R}, \mathbb{R}$ satisfies the right Ore condition for $S$ and $\mathbb{R} S^{-1}=Q\left[x, \sigma^{\prime}, \delta^{\prime}\right]$ ([2], Theorem 7.1.2).

2. If $A$ is a simple right Artinian ring, then $\mathbb{R}=A[x, \sigma, \delta]$ is a principal right ideal ring, where $\sigma$ is an automorphism of $A$ and $\delta$ is a $\sigma$-derivation ([2], Corollary $6.2 .2)$.

3. Suppose $n<\infty$ and $A$ is a ring with $K(A)>n$. If $M$ is a completely faithful Noetherian right $A$-module, such that $K(M)=n$, then $M$ can be generated by $n+1$ elements [7].

4. If $A$ is a simple right Noetherian ring with $K(A)=n$, then any right ideal of $A$ can be generated by $n+1$ elements [7].

5. (i) Let $A$ be a ring with right Krull dimension and $c \in S$ (the $m$-set of regular elements), then $K\left\{(A / c A)_{\mathbb{A}}\right\}<\mathbb{K}\left(A_{\mathbb{A}}\right)([4]$, Lemma 6.3.9 p.189). 
(ii) If $M_{\mathbb{A}}$ is finitely generated, then $K(M) \leq K\left(A_{\mathbb{A}}\right)$ ([4], Lemma 6.2 .5 p.131).

(iii) If $N_{\mathbb{A}}$ is a submodule of $M_{\mathbb{A}^{\prime}}$ then $K(M)=\sup \{K(N), K(M / N)\}([4]$, Lemma 6.2.4 p.180).

(iv) Let $M_{\mathrm{A}}$ have $\mathrm{Krull}$ dimension and also be the sum of submodules each of which has Krull dimension $\leq \alpha$, then $K(M) \leq \alpha$ ([4], Lemma 6.2.14 p.184).

6. Let $I$ be a nonzero $\sigma$-ideal of the $\sigma$-prime right Noetherian ring $A$, then $I \cap S \neq \phi$, where $S$ is the $m$-set of regular elements ([1], proposition I.12 p.I.14).

7. The following are equivalent for a ring $A$, with an automorphism $\sigma$ of $A$ and a $\sigma$-derivation $\delta$ of $A$.

(i) $A$ is right Noetherian.

(ii) $A[x, \sigma, \delta]$ is right Noetherian ([3], Theorem 2.2.15).

Lemma 1. Let $A$ be a prime right Goldie ring and $\mathbb{R}=A[x, \sigma, \delta]$, where $\sigma$ is an automorphism of $A$ and $\delta$ is a $\sigma$-derivation. If $I$ is a right ideal of $R=A[x, \sigma, \delta]$, then $I$ contains an element $g$ such that $I / J=I / g A[x, \sigma, \delta]$ is a torsion right A-module.

Proof. Since, $A$ is a prime right Goldie ring, then $A$ has a right quotient ring $Q$ which is simple Artinian. By \{remark 1(ii)\} the automorphism $\sigma$ of $A$ and the $\sigma$-derivation $\delta$ of $A$ can be extended in a unique manner to $\sigma^{\prime}$ of $Q$ and a $\sigma^{\prime}$ derivation $\delta^{\prime}$ of $Q$. Consider the Ore extension ring $Q\left[x, \sigma^{\prime}, \delta^{\prime}\right]$, then by remark 1(ii) $\} Q\left[x, \sigma^{\prime}, \delta^{\prime}\right]=A S^{-1}\left[x, \sigma^{\prime}, \delta^{\prime}\right]=A[x, \sigma, \delta] S^{-1}$. Using (remark 2) $Q\left[x, \sigma^{\prime}, \delta^{\prime}\right]$ is a principal right ideal ring. Let $\mathbb{I}$ be a right ideal of $\mathbb{R}=A[x, \sigma, \delta]$, then $I_{S}=I Q\left[x, \sigma^{\prime}, \delta^{\prime}\right]=\left\{k s^{-1} \mid k \in I, s \in S\right\}$. Since $I_{S}$ is an ideal in $Q\left[x, \sigma^{\prime}, \delta^{\prime}\right]$, then $I_{\mathbf{S}}=h A[x, \sigma, \delta]_{\mathbf{S}}$, where $h \in I_{\mathbf{S}}$. Since, $S$ satisfies the right Ore condition, then $I_{\mathrm{S}}=g s^{-1} A[x, \sigma, \delta]_{\mathbf{S}}=g A[x, \sigma, \delta]_{\mathbf{S}}$, where $g \in \mathbb{I} \triangle_{r} A[x, \sigma, \delta]$. Let $J=g A[x, \sigma, \delta]$ and consider the right $A$-module $M=I / J$, this is a torsion right $A$-module. Since, $I \subseteq I_{\mathbb{S}}=g A[x, \sigma, \delta]_{\mathbb{S}}=\left\{k s^{-1} \mid k \in I, s \in S\right\}$, then each $i \in I$ can be written as $i=g f$, where $f \in A[x, \sigma, \delta]_{\mathrm{S}}$. Thus $i=g m s_{1}^{-1}$ where $m \in A[x, \sigma, \delta]$ and $s_{1} \in S$. Accordingly, $i s_{1}=g m \in J=g A[x, \sigma, \delta]$ and $M=I / J$ is a torsion 
right $A$-module.

Lemma 2. Let $A$ be a prime right Noetherian ring which satisfies $T(A)=$ $n<\infty$, then the Ore extension ring $R=A[x, \sigma, \delta]$ of $A$ satisfies $r-B n d(n+1)$.

Proof. Let $I$ be a nonzero right ideal of $R$. Since, $A$ is right Noetherian, then $\mathbb{R}$ is right Noetherian by (remark 7 ) and $I=\sum_{i=1}^{k} g_{i} \mathbb{R}$ say $k>n+1$. Using (Lemma 1), there exists a nonzero element $g \in I$ such that $I / J=I / g R$ is a torsion right $A$-module. Therefore, for each $g_{i} \in I$, there exists $r_{i} \in S$ (the $m$ set of regular elements) such that $g_{i} r_{i} \in J$. Noe, if we define $R$-homomorphism,

$$
\begin{aligned}
\phi: \sum_{i=1}^{k} \oplus\left(g_{i} R / g_{i} r_{i} R\right) & \rightarrow \sum_{i=1}^{k} g_{i} R / \sum_{i=1}^{k} g_{i} r_{i} R \quad \text { as } \\
\phi:\left(g_{1} a_{1}+H_{1}, \ldots, g_{k} a_{k}+H_{k}\right) & \longmapsto\left(g_{1} a_{1}+\ldots+g_{k} a_{k}\right)+H, \text { where }
\end{aligned}
$$

$H_{i}=g_{i} r_{i} R$ and $H=\Sigma_{i=1}^{k} g_{i} r_{i} R$, then it is easily verified that $\phi$ is a well defined onto $R$-homomorphism. Also since, $g_{i} r_{i} \in J$ for each $i=1, \ldots, k$, then $g_{i} r_{i} R \subseteq J$ and $\sum_{i=1}^{k} g_{i} r_{i} R \subseteq J$, thus $\Phi: \sum_{i=1}^{k} g_{i} R / \sum_{i=1}^{k} g_{i} r_{i} R \rightarrow \sum_{i=1}^{k} g_{i} R / g R=I / J$, is onto.

Consequently, $\tau: \sum_{i=1}^{k} \oplus_{i} R / g_{i} r_{i} R \rightarrow I / J$, where $\tau=\Phi \circ \phi$ is also onto. Moreover, if we define $R$-homomorphism-:

$\Theta: \sum_{i=1}^{k} \oplus\left(R / r_{i} R\right) \rightarrow \Sigma\left(g_{i} R / g_{i} r_{i} R\right)$ as $\Theta:\left(a_{1}+H_{1}^{\prime}, \ldots, a_{k} H_{k}^{\prime}\right) \longmapsto\left(g_{1} a_{1}+\right.$ $\left.H_{1}, \ldots, g_{k} a_{k}+H_{k}\right)$, where $H_{i}^{\prime}=r_{i} R$, then it is easily shown that $\Theta$ is well defined onto $R$-homomorphism.

Summerizing $I / J$ is the homomorphic image of $\Sigma_{i=1}^{k}{ }^{\oplus} R / r_{i} R$. Since, $A$ satisfies $T(A)=n<\infty$, and $\bigoplus_{i=1}^{k} A / r_{i} A$ is finitely generated torsion right $A$ module, hence $\bigoplus_{i=1}^{k} A / r_{i} A$ can be generated by $n$ elements as $A$-module. Therefore, $\bigoplus_{i=1}^{k} R / r_{i} R$ can also be generated by $n$ elements as $R$-module. Since $\mathbb{I} / J$ is its homomorphic image, then it is generated as an $R$-module by $n$ elements. Hence, $I$ is generated by $n+1$ elements and the Lemma is proved.

The following result shows how can the right (left) Krull dimension [6] play an important role in determining the upper bound of the number of generators of the right (left) ideals in Ore extension rings. 
Proposition 3. Let $A$ be a simple right Noetherian ring and $K\left(A_{\mathbb{A}}\right)=n$, then both $A$ and $R=A[x, \sigma, \delta]$ satisfies $r-B n d(n+1)$.

Proof. Since, $A$ is a simple right Noetherian ring and $\mathbb{K}\left(A_{\mathbb{A}}\right)=n$, then by (remark 4) $A$ satisfies $r$-Bnd $(n+1)$. Also, using (remark 7$) R$ is right Noetherian. Then by the same argument used in (Lemma 1) one can easily check that any nonzero right ideal $I$ of $R$ contains a nonzero element $g$ and the right $R$-submodule $J=g R$ such that $I / J$ is a torsion right $A$-module. Let $I=\sum_{i=1}^{k} a_{i} R$, where $k>n+1$, since $I / J$ is a torsion right $A$-module, then for each $a_{i}$ there exists $r_{i} \in S$ such that $a_{i} r_{i} \in J$. Also, as in (Lemma 2) it can be easily verified that $I / J$ is the homomorphic image of $\Sigma_{i=1}^{k}{ }^{\oplus}\left(A / r_{i} A\right)[x, \sigma, \delta]$. Since, each $r_{i}$ is regular and $K(A)=n$, then by \{remark $\left.5(i)\right\} K\left(A / r_{i} A\right)<n$ for each $i=1, \ldots, k$. Consider the right $A$-module $M=\sum_{i=1}^{k} A / r_{i} A$, since $M$ is a finitely generated $A$-module, then $K(M) \leq n$ by \{remark $5(i i)\}$ and since, $M$ is the sum of submodules each of Krull dimension $<n$, then by \{remark 5(iv) $\} K(M)<n$. Since, $M$ is the homomorphic image of $\bigoplus_{i=1}^{k} A / r_{i} A$ we get that $K\left(\bigoplus_{i=1}^{k} A / r_{i} A\right) \leq K(M)<n$ by $\{$ remark $5($ iii $)\}$. Since. $A$ is simple and $K\left(\bigoplus_{i=1}^{k} A / r_{i} A\right)<n$, then by (remark 3$) \bigoplus_{i=1}^{k} A / r_{i} A$ can be generated by $n$ elements as $A$-module. Conequently, $\bigoplus_{i=1}^{k} A / r_{i} A[x, \sigma, \delta]$ can be generated by $n$ elements as $R$-module. Hence, $I / J$ can be generated by $n$ elements as a homomorphic image of $\bigoplus_{i=1}^{k} A / r_{i} A[x, \sigma, \delta]$. Then $I$ can be generated by $n+1$ elements.

Propossition 4. Let $A$ be a $\sigma$-prime right Noetherian ring satisfies $T(A)=$ $n<\infty$. Then $A$ is $\sigma$-simple.

Proof. Suppose that $A$ is not $\sigma$-simple, then it contains a proper $\sigma$-ideal $p$, take $M$ to be the direct sum of $m$ copies of $A / p$ where $m>1$. Since $A$ is a $\sigma$-prime right Noetherian ring and $p$ is a nonzero $\sigma$-ideal, then by (remark 6 ) $p \cap S \neq \phi$. The regular elements that belong to $p$ annihilate all components of 
$M=(A / p)^{m}$. Thus, $M=(A / p)^{m}$ is a finitely generated torsion right $A$-module which can't be generated by less than $m>n$ elements which contradicts our asssumption. Thus $A$ is a $\sigma$-simple ring.

Lemma 5. Let $A$ be a $\sigma$-prime right Noetherian ring such that $A[x, \sigma]$ satisfies $r-B n d(n)$, then $A$ satisfies $T(A)=n<\infty$ and $A$ is $\sigma$-simple.

Proof. Consider a finitely generated torsion right $A$-module $M=\sum_{i=1}^{m} a_{i} A$, $m>n$. So, for each $a_{i}$ there exists $r_{i} \in S$ (the $m$-set of regular elements) such that $a_{i} r_{i}=0$. Let $\alpha_{i}$ be an $A$-homomorphism: $A \rightarrow a_{i} A$, since $a_{i} r_{i}=0$ then $r_{i} A \subseteq k_{k e r \alpha_{i}}$ for each $i=1, \ldots, m$ and we have an onto $A$-homomorphism: $A / r_{i} A \rightarrow A / k e r \alpha_{i} \simeq a_{i} A$. Now, consider the $A$-homomorphism $\phi: \oplus_{i=1}^{m} A /$ ker $\alpha_{i} \rightarrow \sum_{i=1}^{m} a_{i} A=M$ defined by $\phi:\left(b_{1}+\right.$ ker $\alpha_{1}, \ldots, b_{m}+$ ker $\left.\alpha_{m}\right) \longmapsto \sum_{i=1}^{m} a_{i} b_{i} . \phi$ is well defined, since if $\left(b_{1}+k e r \alpha_{1}, \ldots, b_{m}+\right.$ ker $\left.\alpha_{m}\right)=0$, then each $b_{i} \in$ ker $\alpha_{i}$ (i.e. $\left.a_{i} b_{i}=0\right)$ hence, $\sum_{i=1}^{m} a_{i} b_{i}=0$ and it is clear that $\phi$ is onto. Let $\Phi: \oplus_{i=1}^{m} A / r_{i} A \rightarrow \oplus_{i=1}^{m} A /$ ker $\alpha_{i}$ be an $A$-homomorphism defined by $\Phi:\left(b_{1}+\right.$ $\left.r_{1} A, \ldots, b_{m}+r_{m} A\right) \longmapsto\left(b_{1}+\operatorname{ker} \alpha_{1}, \ldots, b_{m}+\operatorname{ker} \alpha_{m}\right)$ it clear that $\Phi$ is a well defined and onto $A$-homomorphism. So, $M$ is a homomorphic image of $\oplus_{i=1}^{m} A / r_{i} A$. Define an $A$-homomorphism $\mu: \oplus_{i=1}^{m} A / r_{i} A \rightarrow \oplus_{i=1}^{m} A / \sigma^{m-i+1}\left(r_{i}\right) A$ by $\mu:\left(b_{1}+r_{1} A, \ldots, b_{m}+r_{m} A\right) \longmapsto\left(\sigma^{m}\left(b_{1}+r_{1} A\right), \ldots, \sigma\left(b_{m}+r_{m} A\right)\right)=\left(\sigma^{m}\left(b_{1}\right)+\right.$ $\left.\sigma^{m}\left(r_{1} A\right), \ldots, \sigma\left(b_{m}\right)+\sigma\left(r_{m}\right) A\right) \mu$ is well defined since $\mu\left(b_{1}+r_{1} A, \ldots, b_{m}+r_{m} A\right)=$ 0 iff $b_{i} \in r_{i} A, i=1, \ldots, m$ which is equivalent to $\sigma^{m-i+1}\left(b_{i}\right)=\sigma^{m-i+1}\left(r_{i}\right) a^{\prime} \in$ $\sigma^{m-i+1} r_{i} A$, where $a^{\prime} \in A$. Thus, $0=\left(b_{1}+r_{1} A, \ldots, b_{m}+r_{m} A\right) \longmapsto(\overline{0}, \ldots, \overline{0})$ also, $\mu$ is onto. Hence, $\oplus_{i=1}^{m} A / r_{i} A \simeq \oplus_{i=1}^{m} A / \sigma^{m-i+1}\left(r_{i}\right) A=N$. Give $N$ an $A[x, \sigma]$-module structure by defining $N x=0$. Let $I$ be a nonzero right ideal of $A[x, \sigma]$ given by $I=x^{m} A[x, \sigma]+r_{1} x^{m-1} A[x, \sigma]+\ldots+\left(r_{1} \ldots r_{m-1}\right) x A[x, \sigma]$.

Define an $A[x, \sigma]$-homomorphism $\Omega: I \rightarrow N$ as follows $\Omega:\left(x^{m} f_{1}(x)+\right.$ $\left.r_{1} x^{m-1} f_{2}(x)+\ldots+r_{1} \ldots r_{m-1} x f_{m}(x)\right) \longmapsto\left(f_{1}(0)+H_{1}, f_{2}(0)+H_{2}, \ldots, f_{m}(0)+\right.$ $\left.H_{m}\right)$ where $H_{i}=\sigma^{m-i+1}\left(r_{i}\right) A . \Omega$ is a well defined since if $g(x) \in I$, then $g$ can be written as $g(x)=x^{m} \alpha_{0}^{(1)}+r_{1} x^{m-1}\left(\alpha_{0}^{(2)}+x \alpha_{1}^{(2)}\right)+\ldots+r_{1} \ldots r_{m-1} x\left(\alpha_{0}^{m}+\ldots+\right.$ $\left.x^{m-1} \alpha_{m-1}^{(m)}\right)+$ terms of higher degrees, where $\sigma_{j}^{(i)}$ is the coefficient of $x^{j}$ in the polynomial $f_{i}(x)$. Thus $g(x)=x^{m}\left\{\alpha_{0}^{(1)}+\sigma^{m}\left(r_{1}\right) \alpha_{1}^{(2)}+\ldots+\sigma^{m}\left(r_{1}\right) \ldots \sigma^{m}\left(r_{m-1}\right)\right.$ 
$\left.\alpha_{m-1}^{(m)}\right\}+r_{1} x^{m-1}\left\{\alpha_{0}^{(2)}+\sigma^{m-1}\left(r_{2}\right) \alpha_{1}^{(3)}+\ldots+\alpha^{m-1}\left(r_{2} \ldots r_{m-1}\right) \alpha_{m-2}^{(m-1)}\right\}+\ldots+$ $\left(r_{1} \ldots r_{m}\right) x \alpha_{0}^{m}$. But $g(x)=0$ means that $\alpha_{0}^{(1)} \in \sigma^{m} r_{1}(A), \alpha_{0}^{(2)} \in \sigma^{(m-1)}\left(r_{2}\right) A$, $\ldots, \alpha_{0}^{(m)}=0$ which gives that $f_{1}(0)=\alpha_{0}^{(1)} \in H_{1}, f_{2}(0)=\alpha_{0}^{(2)} \in H_{2}, \ldots, f_{m}(0)$ $=\alpha_{0}^{(m)}=0$ i.e. $\Omega g(x)=0$. That $\Omega$ is an $A[x, \sigma]$-module homomorphism follows directly if we notice that $\Omega(g(x) \alpha)=n \alpha$ and $\Omega(g(x) x)=n x=0$ where $\alpha \in A$ and $n=\Omega(g(x))$. Also, it is evident that $\Omega$ is onto. Therefore, $N$ is a homomorphic image of $I$ as $A[x, \sigma]$-module. Since, $A[x, \sigma]$ satisfies $r$ - $\operatorname{Bnd}(n)$, then $I$ can be generated by $n$ elements. Consequently, $N$ is generated as $A[x, \sigma]$ module by $n$ elements. But since, $N x=0$, the same $n$ elements will generate $N$ as an $A$-module. Thus $M$, as a homomorphic image of $N$, is generated by $n$ elements. Consequently, $A$ satisfies $T(A)=n<\infty$ and by (Lemma 4 ) $A$ is $\sigma$-simple.

Now if we put $\delta=0$ in (Lemma 2) then it follows, using the above proposition that

Theorem 6. If $A$ is a prime right Noetherian ring, then the following conditions are equivalent:

1) A. satisfies $T(A)=n<\infty$

2) $A[x, \sigma]$ satisfies $r-B n d(n=1)$.

Proposition 7. Let $A$ be a ring such that $A[x, \sigma]$ satisfies $r-B n d(n)$, then $T(A / p)=n$ for each $\sigma$-prime ideal $p$ of $A$.

Proof. Since $A[x, \sigma]$ satisfies $r-\operatorname{Bnd}(n)$, then $A[x, \sigma]$ is right Noetherian. So, by (remark 7) $A$ is right Noetherian. Since $p$ is a $\sigma$-prime ideal, then $A / p$ is a $\sigma^{\prime}$ prime ring, where $\sigma^{\prime}$ is an automorphism of $A / p$ induced by $\sigma$ and $A / p\left[x, \sigma^{\prime}\right] \simeq$ $A[x, \sigma] / p[x, \sigma]$. Since, $A[x, \sigma]$ satisfies $r$ - $\operatorname{Bnd}(n)$, then $A[x, \sigma] / p[x, \sigma]$ satisfies $r$ $\operatorname{Bnd}(n)$. Hence, $A / p\left[x, \sigma^{\prime}\right]$ satisfies $r-\operatorname{Bnd}(n)$. Using (Lemma 5$) A / p$ satisfies $T(A / p)=n<\infty$.

Corollary 8. Let $A$ be a ring such that $A[x, \sigma]$ satisfies $r-B n d(n)$, then all $\sigma$-prime ideal of $A$ are $\sigma$-maximal. 
Proof. This follows directly from propositions 4 and 7.

I would like to express my gratitude to Professors J. M. Goursaud and M. H. Fahmy, for their helpful suggestions which has improved the results of this paper.

\section{References}

[1] G. Bonnefond, "Thesis University of Poitiers", France 1979.

[2] G. Couchon, "Thesis University of Orsay", France 1977.

[3] A. El-Ahmer, "Thesis University of Poitiers", France 1979.

[4] J. C. McConnell and J. C. Robson, "Non commutative Noetherian rings" A Wiely interscience Publication 1987.

[5] R. Rentschler and P. Gabriel, "Sur la dimension des anneaux et ensemble ordonnes" C. R. Acad. Sci. Paris 265, (1967), 712-715.

[6] J. C. Robson, Cyclic and Faithful objects in quotient categories with application to Noetherian simple or Asano rings, in "Noncummutative ring theory", Lecture Notes in Math. No. 545, Springer-Verlag, 1975.

[7] J. T. Stafford, "Completely faithful modules and ideals of simple Noetherian rings", Bull. Lond. Math. Soc. 8, (1976), 168-173.

[8] J. T. Stafford, "Bounded number of generators of right ideals in poly nomial rings", Comm. in Alg., 8(16) (1980), 1513-1518.

Department of Mathematics, Faculty of Science, Al-AZHAR University, Nasr City, Cairo, Egypt. 